(C) The Authors 2014

\title{
Anthocyanins from fruit juices improve the antioxidant status of healthy young female volunteers without affecting anti-inflammatory parameters: results from the randomised, double-blind, placebo-controlled, cross-over ANTHONIA (ANTHOcyanins in Nutrition Investigation Alliance) study
}

\author{
Sabine Kuntz ${ }^{1 *}$, Clemens Kunz ${ }^{2}$, Johannes Herrmann ${ }^{3}$, Christian H. Borsch ${ }^{2}$, Georg Abel ${ }^{2}$, \\ Bettina Fröhling ${ }^{4}$, Helmut Dietrich ${ }^{4}$ and Silvia Rudloff ${ }^{1}$ \\ ${ }^{1}$ Department of Pediatrics, Justus-Liebig-University Giessen, Giessen, Germany \\ ${ }^{2}$ Institute of Nutritional Science, Justus-Liebig-University Giessen, Wilhelmstrasse 20, D-35392 Giessen, Germany \\ ${ }^{3}$ IT Service Center, Statistical Consulting Service Unit, Justus-Liebig-University Giessen, Giessen, Germany \\ ${ }^{4}$ Geisenheim Research Center, Section of Wine Analysis and Beverage Technology, Geisenheim, Germany \\ (Submitted 31 October 2013 - Final revision received 16 May 2014-Accepted 22 May 2014 - First published online 4 August 2014)
}

\section{Abstract}

Anthocyanins (ACN) can exert beneficial health effects not only through their antioxidative potential but also through modulation of inflammatory parameters that play a major role in CVD. A randomised cross-over study was carried out to investigate the effects of ACN-rich beverage ingestion on oxidation- and inflammation-related parameters in thirty healthy female volunteers. The participants consumed $330 \mathrm{ml}$ of beverages (placebo, juice and smoothie with 8.9 (SD 0.3), 983.7 (sD 37) and 840.9 (sD 10) mg/l ACN, respectively) over $14 \mathrm{~d}$. Before and after each intervention, blood and $24 \mathrm{~h}$ urine samples were collected. Plasma superoxide dismutase (SOD) and catalase activities increased significantly after ACN-rich beverage ingestion $(P<0.001)$, whereas after placebo juice ingestion no increase could be observed. Plasma glutathione peroxidase and erythrocyte SOD activities were not affected. An increase in Trolox equivalent antioxidant capacity could also be observed after juice $(P<0 \cdot 001)$ and smoothie $(P<0 \cdot 01)$ ingestion. The plasma and urinary concentrations of malondialdehyde decreased after ACN-rich beverage ingestion $(P<0 \cdot 001)$, whereas those of 8-OH-2-deoxyguanosine as well as inflammation-related parameters (IL-2, -6, -8 and -10, C-reactive peptide, soluble cluster of differentiation 40 ligand, TNF- $\alpha$, monocyte chemoattractant protein-1 and soluble cell adhesion molecules) were not affected. Thus, ingestion of ACN-rich beverages improves antioxidant enzyme activities and plasma antioxidant capacity, thus protecting the body against oxidative stress, a hallmark of ongoing atherosclerosis.

\section{Key words: Anthocyanins: Antioxidative parameters: Anti-inflammatory parameters: Interventions}

Atherosclerotic vascular disease is the underlying cause of CVD with high morbidity and mortality rates ${ }^{(1)}$. Although the pathophysiological mechanisms underlying atherosclerosis (AS) are not completely understood, it is widely accepted that both oxidative stress and inflammatory stress are key processes involved in the onset and progression of endothelial dysfunction in AS leading to $\mathrm{CVD}^{(2,3)}$. A number of markers that describe oxidative stress and chronic subclinical inflammation at different stages in initiation and progression have been proposed as significant predictors of $\mathrm{AS}^{(4,5)}$. Enhanced generation of harmful reactive oxygen species (ROS) induced by either dysregulated antioxidant enzyme systems or deficiency of antioxidative molecules is associated with oxidative stress leading to the oxidation of target molecules to oxidant-modified molecules. As a consequence, the recruitment and adhesion of monocytes is enhanced; for example, oxidant LDL can increase the secretion of soluble chemotactic molecules such as monocyte chemoattractant protein-1 and IL-8 and enhance the expression of cell adhesion molecules (CAM). The expression of integrins such as intracellular adhesion molecule-1 (ICAM-1) and vascular adhesion molecule-1 (VCAM-1), as well as selectins such as E-selectin, has been found to be enhanced on the surface of activated endothelial cells ${ }^{(6)}$. The progression is further mediated by

Abbreviations: 8-OH-dG, 8-OH-2-deoxyguanosine; ABTS, 2,2'-azinobis(3-ethylbenzothiazoline-6-sulphonic acid); ACN, anthocyanin; ANTHONIA, ANTHOcyanins in Nutrition Investigation Alliance; ARE, antioxidant response element; AS, atherosclerosis; CAM, cell adhesion molecules; CAT, catalase; CRP, C-reactive peptide; GPx, glutathione peroxidase; HPLC-PDA/ESI-MS, HPLC-photodiode array detection/electrospray ionisation MS ICAM-1, intracellular adhesion molecule-1; IQR, interquartile range; MDA, malondialdehyde; Nrf2, nuclear factor erythroid 2-related factor 2; ROS, reactive oxygen species; SOD, superoxide dismutase; TBARS, thiobarbituric acid-reactive substances; TEAC, Trolox equivalent antioxidant capacity; VCAM-1, vascular adhesion molecule-1. 
the transformation of monocytes into foam cells, which in turn secrete ROS and pro-inflammatory cytokines that maintain the chemotactic stimulus for monocytes adhered to the stimulated vascular endothelium. Evidence regarding the linkage between oxidative and inflammatory stress is also derived from studies with at-risk patients. Deficiency of antioxidant enzyme systems promotes oxidative stress (malondialdehyde (MDA) and 8-iso-PGF2 $\alpha$ ) and inflammation (ICAM-1 and E-selectin) leading to endothelial dysfunction ${ }^{(7,8)}$. Moreover, deficiency of superoxide dismutase (SOD) could promote inflammation by enhancing the secretion of TNF- $\alpha$ and adhesion molecules; administration of SOD3 has been found to alleviate Th2-cell-mediated ovalbumin-induced inflammation in mice ${ }^{(9-11)}$. Strategies targeting oxidative as well as inflammatory events in the onset of AS and thus preventing the onset of CVD are still being discussed. Besides other plant compounds, the ingestion of anthocyanins (ACN) present in high amounts in red-and purple-coloured fruits could be a beneficial strategy ${ }^{(12)}$. Numerous in vitro cell and in vivo animal studies have confirmed the high potential of ACN to act as direct ROS scavengers through their polyhydroxylated groups or indirectly by influencing the expression and activities of antioxidant enzyme systems such as SOD and catalase (CAT), thus providing evidence that ACN can act as health-promoting agents ${ }^{(13,14)}$. Recently, the association of the structural characteristics of ACN and their antioxidant capacities with the inhibitory effects on endothelial dysfunction has been established in vitro ${ }^{(15)}$. For example, ACN such as delphinidin exhibit higher ROS-scavenging activities and exert more significant endothelium-protective effects compared with other flavonoids. They can affect the ROS-sensitive NF-кB signalling pathway responsible for the up-regulation of inflammatory cytokine subsets (IL-1 $\beta$ and TNF- $\alpha$ ), acute-phase proteins or CAM. Furthermore, it has been shown that a variety of plant extracts from raspberries, blueberries, blackcurrants and strawberries at physiological concentrations induce the basal NF- $\mathrm{B}$ activity as well as $N F-\kappa B$ activation ${ }^{(16)}$. It has recently been shown that nuclear factor erythroid 2-related factor 2 (Nrf2), together with the ROS-sensitive NF- $\mathrm{BB}$ signalling pathway, is an essential transcription factor that regulates the expression of several antioxidant enzymes including SOD and haem oxygenase- 1 by binding to the antioxidant response element (ARE) ${ }^{(17,18)}$. Similar to their possible interaction with the NF-кB pathway, ACN have been shown to play a crucial role in the activation of the Nrf2-ARE pathway. It has been shown that serumenriched ACN and/or metabolites formed upon the ingestion of ACN induce a significant nuclear accumulation of $\mathrm{Nrf} 2$, as well as the expression of Nrf2-regulated antioxidant and cytoprotective genes ${ }^{(19)}$. Interestingly, it has recently been shown that the metabolites rather than the parental $\mathrm{ACN}$ are responsible for this effect. Phloroglucinol aldehyde, which is known to be a colonic degradation product of ACN, has been shown to significantly increase ARE promoter activity with a concomitant increase in the transcript levels of $\mathrm{Nrf} 2$ or haem oxygenase- $1^{(20)}$.

In spite of the favourable health-promoting effects of $\mathrm{ACN}$, human data are limited and non-distinctive. In some studies,
ACN have been identified as antioxidative and anti-inflammatory molecules due to their potential to suppress the secretion of CAM or chemoattractant cytokines (IL-8 and IL-6) ${ }^{(21-24)}$, and in other studies, a significant increase in the secretion of TNF- $\alpha$ has been observed after blueberry consumption ${ }^{(25,26)}$. As strategies to prevent the onset of AS should be implemented as early as possible, a cross-over intervention study was carried out with young and healthy females to investigate the effects of the ingestion of ACN-rich grape/bilberry juice and smoothie on antioxidative enzymatic and nonenzymatic parameters as well as on cytokine profiles and anti-inflammatory parameters in comparison with those of the ingestion of an ACN-depleted juice as a placebo control.

\section{Subjects and methods}

\section{Study design and study subjects}

A randomised, double-blind, placebo-controlled, cross-over study was conducted to investigate the effects of ACN-rich beverage ingestion in healthy young individuals. The ANTHONIA (ANTHOcyanins in Nutrition Investigation Alliance) study was carried out at the Institute of Nutritional Science, Justus-Liebig-University Giessen (Germany), between April 2010 and August 2010. The present study was conducted according to the guidelines laid down in the Declaration of Helsinki, and the local ethics committee (registration no. 13/10) approved all procedures involving human subjects. Written informed consent was obtained from all subjects. Of the forty-five healthy female students who volunteered, thirty were deemed eligible for the study according to the specific inclusion criteria (3 months without using medications (drugs and antibiotics) and vitamin and mineral supplementation and free from intestinal diseases and CVD). The subjects included in the study were aged between 23 and 27 years (mean 24.6 (SD 1.2) years); their weight ranged between 44 and $78 \mathrm{~kg}$ (mean $59 \cdot 4$ (SD 8.0$) \mathrm{kg}$ ) and their BMI between 18.2 and $27.9 \mathrm{~kg} / \mathrm{m}^{2}$ (mean $21.2(\mathrm{sD} 2 \cdot 3) \mathrm{kg} / \mathrm{m}^{2}$ ). To avoid carry-over effects according to permuted-block randomisation, six groups receiving beverages ((1) placebo, (2) juice and (3) smoothie) in different sequences were generated: $1-2-3 ; 2-3-1$; $3-2-1 ; 3-1-2 ; 1-3-2 ; 2-1-3$. The subjects were assigned in a double-blind fashion to the initial arm of the study of juice, smoothie or placebo intervention. After randomisation, a $10 \mathrm{~d}$ washout period was followed by a $14 \mathrm{~d}$ intervention period and a $4 \mathrm{~d}$ run-out period. The study design is shown in Fig. 1. After an overnight fast, the subjects consumed 0.33 litres of a beverage (juice, smoothie or placebo) daily with breakfast during the intervention period. They were instructed to keep the beverages cool and to avoid exposing them to direct light. Beverage ingestion was monitored by questionnaires after the follow-up visit ( $24 \mathrm{~h}$ after ingestion) to ensure uptake compliance.

Special care was taken to avoid a possible effect of other phenolics in the diet. As phenolic compounds are present in many foodstuffs and beverages, the participants were explicitly counselled before the start of the study to follow a low-phenolic diet during the washout and intervention 

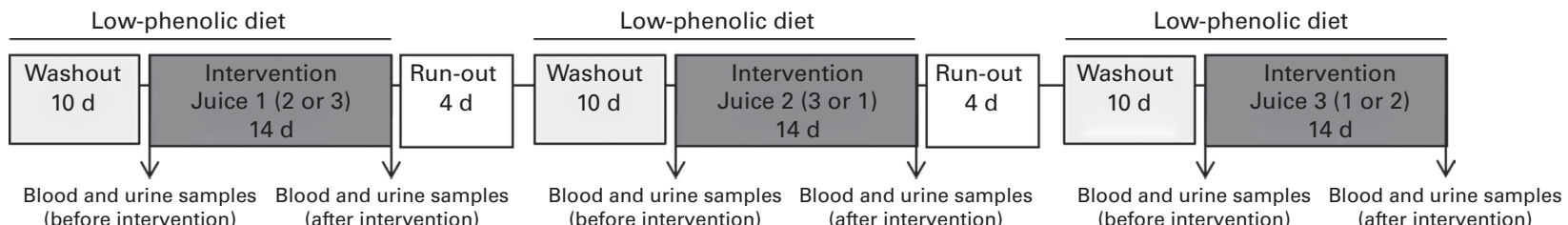

Fig. 1. Design of the ANTHONIA (ANTHOcyanins in Nutrition Investigation Alliance) study. The study participants ( $n 30$ ) were randomly assigned to three beverage intervention groups. They passed three cycles of the intervention (14 d) receiving fruit juice (juice 1), smoothie (juice 2 ) or placebo (juice 3 ). Intervention periods were separated by a $4 \mathrm{~d}$ run-out phase and a $10 \mathrm{~d}$ washout phase. Before (day 0 ) and after (day 14) the intervention, blood and $24 \mathrm{~h}$ urine samples were collected and processed for biochemical analyses. After a $14 \mathrm{~d}$ intervention, the participants crossed over to the alternate beverage. All participants completed the study.

periods and maintain their usual physical activity. In addition, they were given a list categorising foodstuffs, including beverages, into 'not being allowed', 'quantitatively limited' and 'allowed'. Foodstuffs were categorised according to their ACN content based on data from the USDA Database for the Flavonoid Content of Selected Foods (Release 3.1 (December 2013); http://www.ars.usda.gov/) or the database on polyphenol contents in foods (Polyphenol-Explorer, Release 3.0 (http://www.phenol-explorer.eu/compounds); Table 1). The participants were instructed to keep daily dietary records throughout the study, not only to document their daily dietary intake, but also to ascertain their compliance with dietary instructions. Food intake estimated from daily dietary records was analysed using the DGE-PC professional software (version 4.1.0.043). The baseline characteristics of the study population are given in Table 2 .

\section{Source of anthocyanin-rich (juice and smoothie) and anthocyanin-depleted (placebo) beverages}

Juice, smoothie and placebo juice were produced at the Geisenheim Research Center (Section of Wine Analysis and Beverage Technology) and prepared from an 80:20 mixture of red grapes (grape varieties Dakapo and Accent) and bilberries (Vaccinium myrtillus). Briefly, grapes were extracted in a press, and the resulting juice was separated, blended with bilberry juice from a concentrate, pasteurised and hot-filled into 0.33-litre brown glass bottles. The smoothie was prepared by blending the juice with $20 \%$ bilberry purée instead of bilberry juice. The placebo juice was obtained by passing the grape juice through SP70 absorber resin (Resindion S.r.l.). Juice and placebo juice samples were analysed directly after membrane filtration $(0.45 \mu \mathrm{m})$ for basic analytical parameters such as concentrations of total phenolics, concentrations of ACN and Trolox equivalent antioxidant capacity (TEAC) as described elsewhere ${ }^{(27,28)}$. Smoothie samples were extracted twice for HPLC and TEAC analyses with $80 \%$ methanol. Using $10 \mathrm{ml}$ of $80 \%$ methanol, $5 \mathrm{~g}$ of smoothie samples were extracted twice ultrasound-assisted for $30 \mathrm{~min}$. Supernatants were collected and mixed with $80 \%$ methanol after the second extraction. The samples had a $\mathrm{pH}$ of 3 , so the extraction solution was not acidified. Chromatographic analysis of $\mathrm{ACN}$ in juice and placebo juice samples was performed via HPLC/photodiode array detection/electrospray ionisation MS (HPLC-PDA/ESI-MS) using a Thermo Finnigan Surveyor HPLC system coupled to a Thermo Finnigan LCQ Advantage
Max mass spectrometer equipped with an electrospray ionisation source and an ion trap mass analyser. For ACN analysis, the mass spectrometer was operated in the positive mode. Chromatographic separation was achieved on a Reprosil Pur 120 ODS-3 column $(125 \times 2 \mathrm{~mm}$ inner diameter, $5 \mu \mathrm{m}$; Dr. Maisch $\mathrm{GmbH}$ ) at a flow rate of $0.2 \mathrm{ml} / \mathrm{min}$ at $40^{\circ} \mathrm{C}$. Water-formic acid (95:5, v/v) as solvent A and methanol as solvent $\mathrm{B}$ were used at a linear gradient from 10 to $40 \% \mathrm{~B}$ in $18 \mathrm{~min}$. The composition of the ACN-depleted placebo juice and the ACN-rich juice and smoothie is given in Table 3 .

All beverages were matched for fructose content, as it is known to be a confounding variable related to the plasma antioxidant capacity. Beverages were not matched for vitamin $\mathrm{C}$ content as it is low, which has been shown to not significantly affect plasma vitamin levels ${ }^{(28-31)}$. ACN were analysed using HPLC-PDA/ESI-MS, and their content is summarised in Table 4. Quantification was carried out in duplicate using peak areas detected at $520 \mathrm{~nm}$ and based on external calibration using the reference substance malvidin-3-glucoside $\left(0 \cdot 1-100 \mathrm{mg} / \mathrm{l}\right.$; linearity of calibration, $r^{2}$ 0.9999). For malvidin-3-glucoside, the limit of detection was $0.01 \mathrm{mg} / 1$ and the limit of quantification was $0.04 \mathrm{mg} / \mathrm{l}$. The ACN analysis was carried out in duplicate.

\section{Chemicals}

All chemicals were of analytical grade: TCA (ACS reagent, $\geq 99 \%$ ), thiourea (puriss. p.a. (purrissimum pro analysis), ACS reagent, $\geq 99.0 \%$ ), Cu(II) sulphate hydrate (98\%), 2,4-dinitrophenylhydrazine (puriss. p.a., $\geq 99 \cdot 0 \%$ (HPLC)), and water CHROMASOLV $^{\circledR}$ (for HPLC) were obtained from SigmaAldrich and sulphuric acid (98\%) was obtained from Carl Roth GmbH \& Co KG.

\section{Measurement of plasma oxidative and inflammatory biomarkers}

Before (day 0) and after (day 14) the intervention, blood and $24 \mathrm{~h}$ urine samples were collected to measure oxidative biomarkers (SOD, CAT, glutathione peroxidase (GPx), TEAC, MDA and 8-OH-2-deoxyguanosine (8-OH-dG)) and plasma inflammatory biomarkers (cytokines (IL-2, IL-6, IL-10 and TNF- $\alpha$ ), chemokines (IL-8 and monocyte chemoattractant protein-1), CAM (soluble cluster of differentiation 40 ligand, soluble VCAM-1 and soluble ICAM-1) and acute-phase proteins (C-reactive peptide; CRP)). Blood samples were 


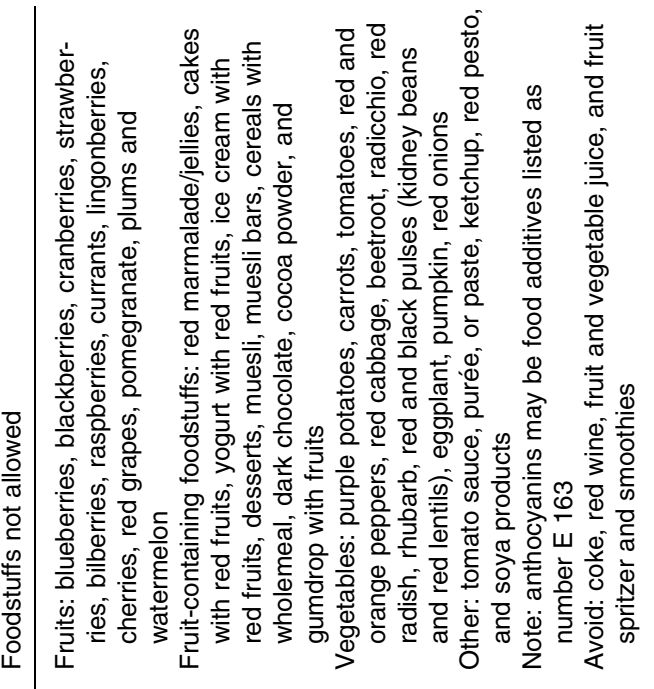

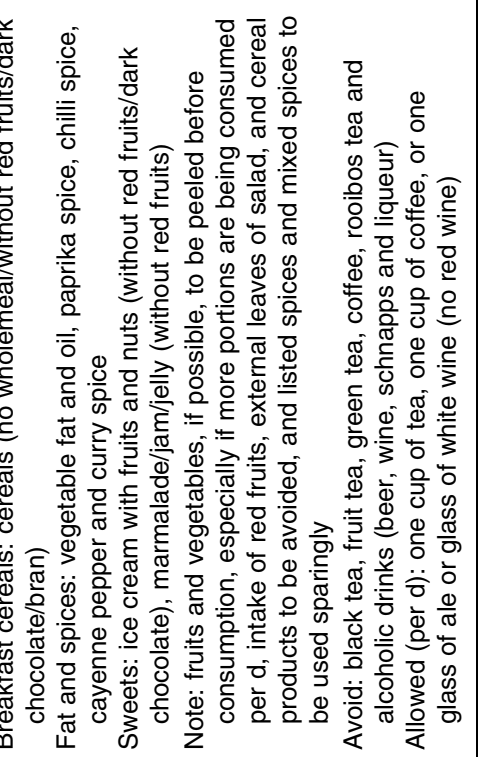

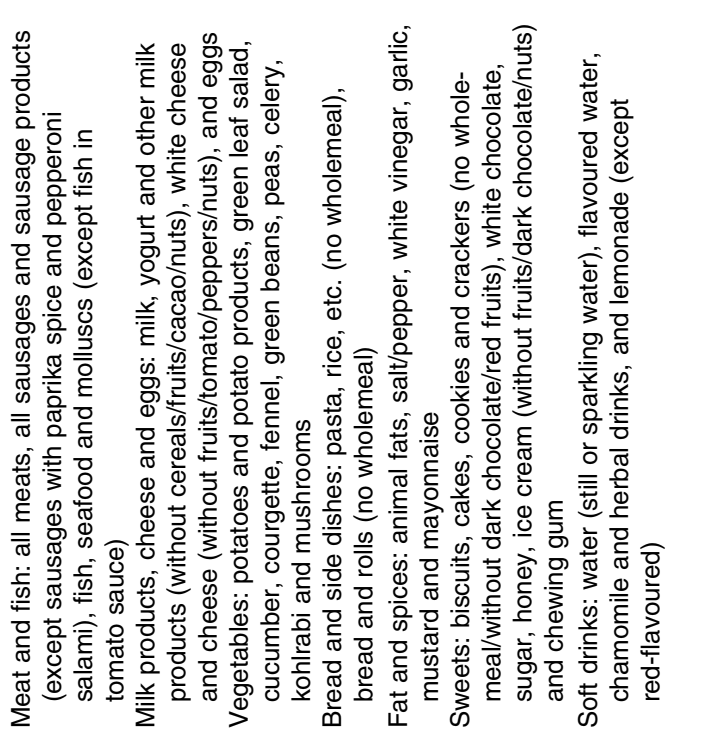

drawn by venepuncture into tubes with EDTA anticoagulant (Sarstedt AG \& Co.). Blood samples collected $5 \mathrm{~h}$ after juice ingestion were immediately centrifuged ( $1200 \boldsymbol{g}$ for $15 \mathrm{~min}$ at $\left.4^{\circ} \mathrm{C}\right)$ to separate plasma, and the supernatant was divided into aliquots and stored in plastic tubes at $-80^{\circ} \mathrm{C}$ until analysis. For the determination of erythrocyte SOD activity, plasma was removed and then erythrocytes were haemolysed by addition of an equal volume of ice-cold water (1:1, v/v) and mixed well. After centrifugation for $5 \mathrm{~min}$ at $1500 \mathrm{~g}$, lysates were stored at $-80^{\circ} \mathrm{C}$. These haemolysates were diluted to 1:20 for the assessment of erythrocyte SOD activity. Erythrocyte SOD activity was assessed as protein concentration in $\mathrm{U} / \mathrm{mg}$ protein. Protein concentration was determined photometrically using the Pierce ${ }^{\mathrm{TM}}$ BCA Protein Assay Kit according to the manufacturer's instructions (Thermo Fisher Scientific).

SOD, CAT and GPX activities were determined by colorimetric methods according to the manufacturer's instructions (Cayman Chemical Company) in a microplate reader (DigiScan 340 and 400; Asys Hitech GmbH).

The amount of water and lipid-soluble antioxidants in plasma was determined using the ABTS (2,2'-azino-di-3-ethylbenzthiazoline sulphonate) assay (Cayman Chemical Company). The generation of $\mathrm{ABTS}^{+}$from ABTS by metmyoglobin is inhibited by antioxidants, resulting in the reduction of absorbance at $405 \mathrm{~nm}$. The capacity of antioxidants to reduce $\mathrm{ABTS}^{+}$generation was compared with that of Trolox and quantified as Trolox equivalents (mmol/1 TEAC) ${ }^{(32-34)}$.

The measurement of lipid peroxidation levels in plasma is based on the fact that MDA, a naturally occurring product of lipid peroxidation, reacts with thiobarbituric acid under high temperature and acidic conditions (Cayman Chemical Company). The resulting adducts (MDA-thiobarbituric acid) can be determined fluorimetrically (excitation $530 \mathrm{~nm} / \mathrm{emission}$ $550 \mathrm{~nm}$ ). The concentration of MDA was calculated using a standard curve, and it is expressed as $\mu \mathrm{mol} / \mathrm{ml}$ TBARS (thiobarbituric acid-reactive substances) in plasma or $\mu \mathrm{mol} / \mathrm{g}$ creatinine in urine.

The concentration of $8-\mathrm{OH}-\mathrm{dG}$ as a marker of oxidative DNA damage induced by ROS was determined using a competitive enzyme-linked sandwich immunoassay (Cayman Chemical Company) for free $8-\mathrm{OH}-\mathrm{dG}$. Concentrations in urine were normalised to those of creatinine and are expressed as $\mathrm{pg} / \mathrm{g}$ creatinine.

Urinary creatinine concentrations were determined by the Jaffé reaction according to the manufacturer's instructions (http://www.rndsystems.com/pdf/KGE005.pdf). The colour intensity of the creatinine-picrate complex at $490 \mathrm{~nm}$ corresponds to the concentration of creatinine in the sample (R\&D Systems) $)^{(35-37)}$.

The plasma concentrations of soluble ICAM, soluble VCAM-1, soluble E-selectin, IL-2, IL-6, IL-8, IL-10, TNF- $\alpha$, high-sensitive CRP, soluble CD40 ligand (sCD40L) and monocyte chemoattractant protein-1 were measured using commercial ELISA according to the manufacturer's instructions (R\&D Systems).

The plasma concentration of vitamin $\mathrm{C}$ was measured spectrophotometrically according to the method of Lowry et $a l^{(38-40)}$. Briefly, the plasma concentrations of total vitamin $\mathrm{C}$ (sum of ascorbic acid and dehydroascorbic acid) were 
Table 2. Baseline characteristics of the study population*

(Mean values and standard deviations, $n 30$ )

\begin{tabular}{|c|c|c|c|c|}
\hline \multirow[b]{3}{*}{ Variables } & \multicolumn{4}{|c|}{ Intervention period (3 months) } \\
\hline & \multicolumn{2}{|c|}{ Before intervention } & \multicolumn{2}{|c|}{ After intervention } \\
\hline & Mean & SD & Mean & SD \\
\hline Age (years) & $24 \cdot 0$ & $1 \cdot 2$ & $24 \cdot 3$ & $1 \cdot 2$ \\
\hline Weight (kg) & $59 \cdot 4$ & 8.0 & $59 \cdot 0$ & $7 \cdot 2$ \\
\hline BMI $\left(\mathrm{kg} / \mathrm{m}^{2}\right)$ & $21 \cdot 2$ & $2 \cdot 3$ & $21 \cdot 1$ & 1.9 \\
\hline \multicolumn{5}{|l|}{ Dietary intake per d } \\
\hline Energy (kcal/d) & 1624 & 591 & 1950 & 439 \\
\hline Energy $(\mathrm{kJ} / \mathrm{d})$ & 6795 & 2473 & 8159 & 1837 \\
\hline Fat $(\mathrm{g} / \mathrm{d})$ & $66 \cdot 5$ & $28 \cdot 6$ & $66 \cdot 4$ & $25 \cdot 3$ \\
\hline Carbohydrates (g/d) & 240 & 60 & 267 & 56 \\
\hline Dietary fibre $(\mathrm{g} / \mathrm{d})$ & $18 \cdot 0$ & $6 \cdot 0$ & $17 \cdot 6$ & 5.6 \\
\hline Protein $(\mathrm{g} / \mathrm{d})$ & $58 \cdot 6$ & $22 \cdot 2$ & $55 \cdot 1$ & $20 \cdot 1$ \\
\hline Retinol equivalents $(\mu \mathrm{g} / \mathrm{d})$ & 807 & 394 & 617 & 277 \\
\hline$\beta$-Carotene $(\mu \mathrm{g} / \mathrm{d})$ & 2050 & 2010 & 1161 & 1029 \\
\hline Vitamin $E(\mu \mathrm{g} / \mathrm{d})$ & 7058 & 6375 & 8640 & 6323 \\
\hline Vitamin C $(\mathrm{mg} / \mathrm{d})$ & $61 \cdot 7$ & $56 \cdot 1$ & $72 \cdot 9$ & 30.2 \\
\hline
\end{tabular}

* Dietary records were analysed using the DGE-PC professional software (version 4.1.0.043).

measured after protein precipitation. Duplicate aliquots of supernatant were added to a 2,4-dinitrophenylhydrazone/

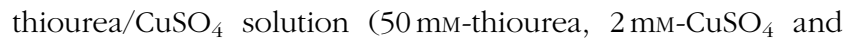
$150 \mathrm{~mm}$-dinitrophenylhydrazine in $9 \mathrm{M}-\mathrm{H}_{2} \mathrm{SO}_{4}$ ). The dehydroascorbic acid formed from the oxidation of ascorbic acid by $\mathrm{Cu}$ was quantified spectrophotometrically as the 2,4-dinitrophenylhydrazone derivative at $550 \mathrm{~nm}$. Ascorbic acid standards were prepared in 5\% TCA, and results are expressed as $\mathrm{mg} / \mathrm{l}$.

\section{Statistical analyses}

Data obtained from participants who completed all phases of the study protocol ( $n$ 30) were analysed. All analyses were carried out in duplicate and intra-assay coefficients of assays were below $10 \%$. The outcome was evaluated according to the differences in antioxidative and anti-inflammatory parameters of the placebo, juice and smoothie treatment groups before (day 0) and after (day 14) the intervention. The data were also tested for covariates (age, height, weight, BMI, vegetarian, smoking and plasma vitamin C concentrations) using a univariate general linear model (ANCOVA). If significant interactions were observed, data obtained before or after the intervention were analysed using two-way repeated-measures ANOVA models. If no significant effect was observed, beforetreatment $v$. after-treatment data of the groups were analysed using two-tailed paired $t$ test and data that were not normally distributed were analysed using Wilcoxon's signed-rank test. The normality of continuous variables was assessed using the Kolmogorov-Smirnov normality test with Lilliefors correction with a $P$ value $<0 \cdot 05$. Rank or log transformations were performed on some variables before analysis to meet parametric analysis assumptions. Data are expressed as means and standard deviations or as medians and interquartile ranges (IQR; 25th-75th percentiles). Although the study design reduced carry-over effects, the possible carry-over effect was determined by testing a period $\times$ treatment interaction term in the general linear models. If no effects were observed, the different intervention periods would be seen independent. The statistical IBM software SPSS for Windows (version 19.0.0) was used for data analysis.

Table 3. Composition of the anthocyanin-depleted placebo and anthocyanin-rich beverages (juice and smoothie)* (Mean values and standard deviations, $n$ 2)

\begin{tabular}{|c|c|c|c|c|c|c|}
\hline & \multicolumn{2}{|c|}{ Placebo } & \multicolumn{2}{|c|}{ Juice } & \multicolumn{2}{|c|}{ Smoothie } \\
\hline & Mean & SD & Mean & SD & Mean & SD \\
\hline Extract $(g / l)$ & 177 & 0 & 206 & 0 & 208 & 0 \\
\hline Sugar-free extract $(\mathrm{g} / \mathrm{l})$ & $22 \cdot 8$ & 0.0 & 31.5 & 0.0 & $36 \cdot 1$ & 0.0 \\
\hline Reducing sugar (g/l) & 155 & 2 & 175 & 5 & 172 & 2 \\
\hline Glucose $(\mathrm{g} / \mathrm{l})$ & $76 \cdot 2$ & 1.0 & $84 \cdot 6$ & 0.5 & $85 \cdot 6$ & 0.3 \\
\hline Fructose (g/l) & 78.4 & 0.1 & 89.2 & 0.2 & 88.6 & 0.3 \\
\hline Sucrose $(\mathrm{g} / \mathrm{l})$ & ND & & ND & & ND & \\
\hline Dietary fibre $(\mathrm{g} / \mathrm{l})$ & 4.00 & 0.40 & 7.00 & 0.90 & 11.0 & 0.6 \\
\hline $\mathrm{pH}$ & 3.56 & 0.3 & 3.49 & 0.7 & 3.43 & 1.8 \\
\hline Acids $(g / l)$ & 5.51 & 0.02 & $7 \cdot 30$ & 0.03 & 7.91 & 0.04 \\
\hline Ascorbic acid (mg/l) & $22 \cdot 0$ & 0.2 & 102 & 1 & 118 & 1 \\
\hline Tartaric acid $(\mathrm{g} / \mathrm{l})$ & 0.03 & 0.00 & $3 \cdot 6$ & $0 \cdot 1$ & 3.8 & 0.1 \\
\hline L-Lactic acid $(\mathrm{g} / \mathrm{l})$ & 0.04 & 0.00 & 0.09 & 0.00 & 0.08 & 0.00 \\
\hline L-Malic acid (g/l) & 2.95 & 0.01 & 3.56 & 0.02 & 3.86 & 0.05 \\
\hline Acetic acid (g/l) & 0.04 & 0.00 & 0.10 & 0.00 & 0.09 & 0.00 \\
\hline Ethanol $(\mathrm{g} / \mathrm{l})$ & ND & & 0.67 & 0.02 & 0.73 & 0.04 \\
\hline $\mathrm{Ca}(\mathrm{mg} / \mathrm{l})$ & $82 \cdot 3$ & $3 \cdot 0$ & $94 \cdot 7$ & $2 \cdot 7$ & $96 \cdot 6$ & $2 \cdot 8$ \\
\hline $\mathrm{K}(\mathrm{mg} / \mathrm{l})$ & 1938 & 28 & 2010 & 24 & 2130 & 11 \\
\hline $\mathrm{Mg}(\mathrm{mg} / \mathrm{l})$ & 88.4 & 1.4 & $95 \cdot 6$ & 0.2 & $95 \cdot 3$ & $1 \cdot 0$ \\
\hline Total phenolics (mg/l) & 179 & 1 & 3227 & 3 & 3435 & 2 \\
\hline TEAC $(\mathrm{mmol} / \mathrm{l})$ & $1 \cdot 10$ & 0.00 & 33.2 & $1 \cdot 0$ & $27 \cdot 2$ & 0.2 \\
\hline Monomeric index & 0.51 & 0.01 & $7 \cdot 84$ & $0 \cdot 10$ & 13.6 & 0.1 \\
\hline
\end{tabular}

ND, not detectable; TEAC, Trolox equivalent antioxidant capacity.

* Juice samples were analysed for standard parameters: TEAC and vitamin C (reductometrically); anthocyanins (HPLC-photodiode array detection/electrospray ionisation MS); total phenolics (Folin reaction, using (+)-catechin as a standard). More details are given in the 'Materials and methods' section. 
Table 4. Anthocyanins present in the beverages (anthocyanin-depleted placebo, anthocyanin-rich juice and smoothie)* (Mean values and standard deviations, $n 2$ )

\begin{tabular}{|c|c|c|c|c|c|c|}
\hline & \multicolumn{2}{|c|}{ Placebo } & \multicolumn{2}{|c|}{ Juice } & \multicolumn{2}{|c|}{ Smoothie } \\
\hline & Mean & SD & Mean & SD & Mean & SD \\
\hline Peonidin-3,5-diglucoside (mg/ml) & ND & & 5.91 & 0.48 & 8.00 & $0 \cdot 10$ \\
\hline Cyanidin-3,5-diglucoside (mg/ml) & ND & & ND & & 4.95 & $0 \cdot 11$ \\
\hline Delphinidin-3-galactoside (mg/ml) & ND & & $9 \cdot 32$ & 0.14 & $43 \cdot 1$ & 1.9 \\
\hline Delphinidin-3-glucoside (mg/ml) & ND & & $67 \cdot 4$ & 1.1 & 105 & 3 \\
\hline Cyanidin-3-galactoside/peonidin derivate $(\mathrm{mg} / \mathrm{ml})$ & ND & & 13.4 & $0 \cdot 1$ & $30 \cdot 2$ & 0.9 \\
\hline Delphinidin-3-arabinoside (mg/ml) & ND & & $10 \cdot 9$ & 0.1 & $30 \cdot 9$ & $1 \cdot 1$ \\
\hline Cyanidin-3-glucoside $(\mathrm{mg} / \mathrm{ml})$ & 0.24 & 0.02 & $41 \cdot 1$ & 0.5 & 58.4 & $2 \cdot 1$ \\
\hline Petunidin-3-galactoside (mg/ml) & 0.38 & 0.03 & ND & & 11.9 & 0.5 \\
\hline Cyanidin-3-arabinoside (mg/ml) & ND & & $12 \cdot 0$ & 0.2 & $18 \cdot 6$ & 0.6 \\
\hline Petunidin-3-glucoside $(\mathrm{mg} / \mathrm{ml})$ & ND & & $69 \cdot 8$ & 1.4 & $87 \cdot 0$ & 3.3 \\
\hline Peonidin-3-galactoside $(\mathrm{mg} / \mathrm{ml})$ & 0.77 & 0.04 & ND & & 4.45 & 0.01 \\
\hline Peonidin-3-glucoside $(\mathrm{mg} / \mathrm{ml})$ & ND & & 165 & 2 & 163 & 6 \\
\hline Malvidin-3-glucoside (mg/ml) & 1.73 & 0.07 & 273 & 4 & 274 & 10 \\
\hline Malvidin-3-arabinoside (mg/ml) & ND & & $4 \cdot 24$ & 0.26 & 5.52 & $0 \cdot 10$ \\
\hline Delphinidin-3-(6'-O-acetyl)-glucoside $(\mathrm{mg} / \mathrm{ml})$ & ND & & $5 \cdot 15$ & 0.20 & $6 \cdot 50$ & 0.67 \\
\hline Cyanidin-3-(6'-O-acetyl)-glucoside $(\mathrm{mg} / \mathrm{ml})$ & ND & & ND & & 2.56 & 0.54 \\
\hline Petunidin-3-(6'-O-acetyl)-glucoside $(\mathrm{mg} / \mathrm{ml})$ & ND & & $10 \cdot 9$ & 0.0 & 8.46 & 1.32 \\
\hline Peonidin-3-(6'-O-acetyl)-glucoside ( $\mathrm{mg} / \mathrm{ml})$ & ND & & $18 \cdot 3$ & 0.1 & 14.7 & $1 \cdot 2$ \\
\hline Malvidin-3-(6'-O-acetyl)-glucoside $(\mathrm{mg} / \mathrm{ml})$ & $2 \cdot 84$ & 0.04 & $51 \cdot 7$ & 0.7 & $46 \cdot 4$ & $2 \cdot 1$ \\
\hline Petunidin-3-(6'-p-coumaryl)-glucoside $(\mathrm{mg} / \mathrm{ml})$ & ND & & $15 \cdot 3$ & 0.1 & $12 \cdot 7$ & $0 \cdot 8$ \\
\hline Malvidin-3-(6'-p-coumaryl)-glucoside $(\mathrm{mg} / \mathrm{ml})$ & $3 \cdot 19$ & 0.02 & $66 \cdot 6$ & 0.3 & $46 \cdot 5$ & 1.9 \\
\hline Sum $(\mathrm{mg} / \mathrm{ml})$ & 8.97 & 0.34 & 840 & 10 & 983 & 37 \\
\hline
\end{tabular}

ND, not detectable.

* Beverages were analysed by HPLC-photodiode array detection/electrospray ionisation MS. More details are given in the 'Materials and methods' section.

\section{Results}

ACN-rich beverages in two different preparations (juice and smoothie) were given to healthy female volunteers over an intervention period of $14 \mathrm{~d}$ to investigate the possible effects of food matrix on the antioxidant and anti-inflammatory parameters of the beverages. An ACN-depleted placebo juice was also used to eliminate the effect of other compounds in the beverages according to the study protocol shown in Fig. 1. The baseline characteristics of the study population are summarised in Table 2. No significant changes were observed during the whole study period.

\section{Antioxidant capacity and beverage composition}

As shown in Tables 3 and 4, the ACN-rich fruit beverages had a very high antioxidant activity (TEAC value) and a high concentration of total phenolics and $\mathrm{ACN}$ in comparison with the ACN-depleted juice. The beverages differed in their polyphenolic composition, which is summarised in Table 4. Malvidin-3-glucoside and peonidin-3-glucoside were identified as the most prominent $\mathrm{ACN}$ followed by petunidin-3-glucoside, malvidin-3-(6'-O-acetyl)-glucoside and cyanidin-3-glucoside. In comparison with the ACN-rich beverages, the ACN-depleted placebo juice contained only minor amounts of the ACN listed in Table 4. Because ascorbic acid was present only in moderate amounts (mean $<118$ (SD 1) $\mathrm{mg} / \mathrm{l}$ ), it did not seem to make a major contribution to the antioxidant properties of the study juice. The mean plasma vitamin $\mathrm{C}$ value at the beginning of the study was
11.8 (sD $2 \cdot 7) \mathrm{mg} / \mathrm{l}$ and did not significantly differ from that recorded at the end of the study (mean 11.3 (sD 2.7) $\mathrm{mg} / \mathrm{l}$ ).

\section{Effect of anthocyanin-rich beverages on antioxidative biomarkers in plasma, erythrocytes and urine}

The ingestion of ACN-rich beverages improved the antioxidative status of the participants. Fig. 2 shows the activities of antioxidant enzymes such as SOD, CAT and GPx in plasma and erythrocytes. A significant increase in plasma SOD activity of about 6\% (median 12.95 (IQR 9.71-16.17) v. 13.73 (IQR 11.95-19.65)) after $14 \mathrm{~d}$ of juice intervention or of about $21 \%$ (median 13.17 (IQR 9.88-16.46) v. $15.98 \quad$ (IQR 11.94-18.88)) after smoothie intervention was observed, whereas no change was observed after placebo juice intervention (median 13.78 (IQR 10.33-17.23) v. 13.64 (IQR 10.64-17.01); Fig. 2(a)). In contrast to plasma SOD activity, erythrocyte SOD activity was not affected by $14 \mathrm{~d}$ of juice, smoothie or placebo intervention (Fig. 2(b)). Similar to plasma SOD activity, plasma CAT activity was significantly increased after the ingestion of ACN-rich beverages (median 4.29 (IQR 3.22-5.36) v. 5.05 (IQR 3.76-6.31) ( $P<0.001)$ after juice ingestion and median 4.31 (IQR 3.23-5.39) v. 5.33 (IQR 3.40-6.66) $(P<0.001)$ after smoothie ingestion). After $14 \mathrm{~d}$ of placebo juice intervention, CAT activity remained unchanged (Fig. 2(c)). In contrast to the observations for SOD and CAT activities, no significant differences were found in plasma GPx activity after the ingestion of both ACN-rich juice and smoothie (Fig. 2(d)). 
The antioxidant capacity of plasma samples was estimated as TEAC comprising the antioxidative capacity of both lipophilic and hydrophilic compounds (Table 5). When comparing the median TEAC values of the placebo group with those of the ACN-rich smoothie and juice groups, an increase of approximately $10 \%$ from $2 \cdot 18$ (IQR $1.64-2.73$ ) to $2 \cdot 48$ (IQR $1 \cdot 86-3 \cdot 10) \mathrm{mmol} / \mathrm{ml}(P<0 \cdot 001)$ was observed after $14 \mathrm{~d}$ of daily juice intervention. After smoothie intervention, the increase in TEAC values was found to be in a similar range (median 2.20 (IQR $1.65-2.75$ ) to 2.43 (IQR $1 \cdot 82-3.03) \mathrm{mmol} / \mathrm{ml} ; P<0 \cdot 01)$.

Lipid peroxidation levels were determined via the formation of TBARS in plasma and urine after $14 \mathrm{~d}$ of placebo, juice and smoothie interventions (Table 5). A significant decrease was observed in plasma and urinary TBARS levels after the ingestion of both ACN-rich juice $(P<0.001)$ and ACN-rich smoothie $(P<0.001)$. In both cases, the reduction was in a similar range ( 4 and $6 \%$, respectively). In addition, no changes were observed in urinary 8-OH-dG concentrations, either on ingesting the juice $(P=0 \cdot 178)$ or smoothie $(P=0 \cdot 285)$ or on ingesting the placebo juice $(P=0 \cdot 395)$.

\section{Effect of anthocyanin-rich beverages on anti-inflammatory biomarkers in plasma}

We aimed to investigate whether the ingestion of ACN-rich beverages had an effect on inflammatory parameters. Table 6 summarises the plasma concentrations of cytokines and soluble CAM at baseline and after $14 \mathrm{~d}$ of juice intervention. Independent of the kind of intervention used and in contrast to the antioxidative effects, inflammatory biomarkers in plasma were found to remain unchanged throughout the study.

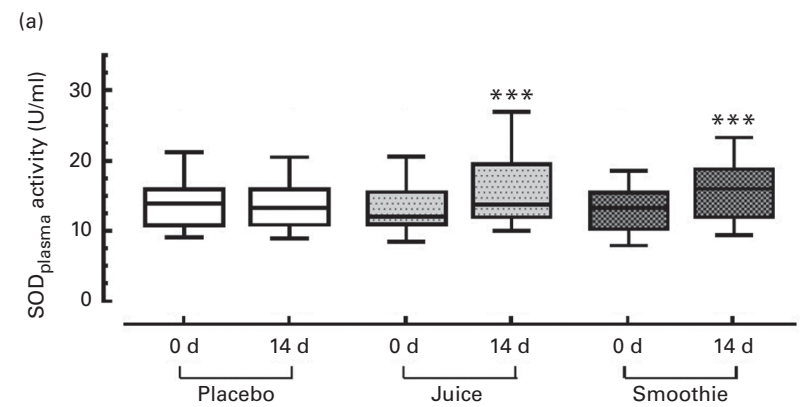

(b)

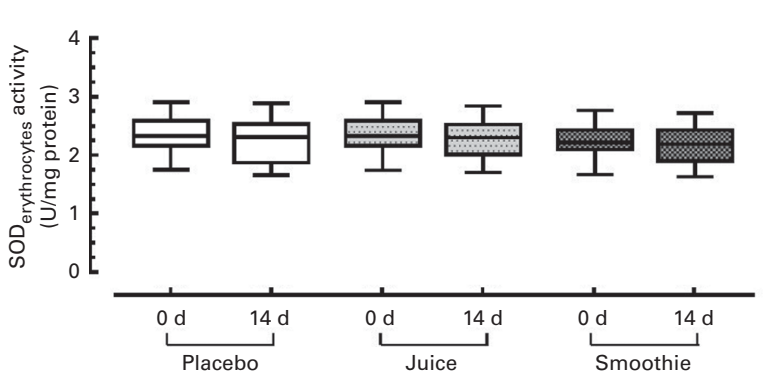

\section{Discussion}

Data from human studies concerning the potential of ACN to reduce the incidence of oxidation- and inflammation-related diseases are very limited and investigations in terms of atherogenesis have just begun. We aimed to investigate whether the ingestion of ACN-rich beverages in two preparations (juice and smoothie) would influence the antioxidative parameters and peripheral markers of endothelial cell activation associated with inflammation in a double-blind, placebocontrolled, cross-over intervention study in healthy young females. The major finding of the present study was that the daily intake of about $277.5 \mathrm{mg}$ (juice) or $324.6 \mathrm{mg}$ (smoothie) of ACN from a mixture of red grape juice and bilberry juice or purée (80:20) over $14 \mathrm{~d}$ improved the antioxidative status of the volunteers determined by means of both non-enzymatic and enzymatic parameters, independently of the juice preparation. The plasma antioxidant capacity, measured as TEAC, was significantly increased when compared with that observed in the placebo group concomitant with reduced plasma and urinary TBARS levels, but there were no effects on urinary 8-OH-dG concentrations. Published data concerning an improvement of antioxidant activity with concomitant effects on target molecules after ACN ingestion are scarce and inconsistent, not least because of the differences in intervention durations and ingested amounts of ACN from juices or berries and in food matrices as well as due to the presence of other polyphenols ${ }^{(41)}$. Although cyanidin-3-glucoside was the major ACN investigated in most studies, the juice or smoothie preparations that we used contained $80 \%$ red grapes and $20 \%$ bilberries with malvidin-3-glucsoide and peonidin-3-glucoside as the major ACN. In comparison with (c)
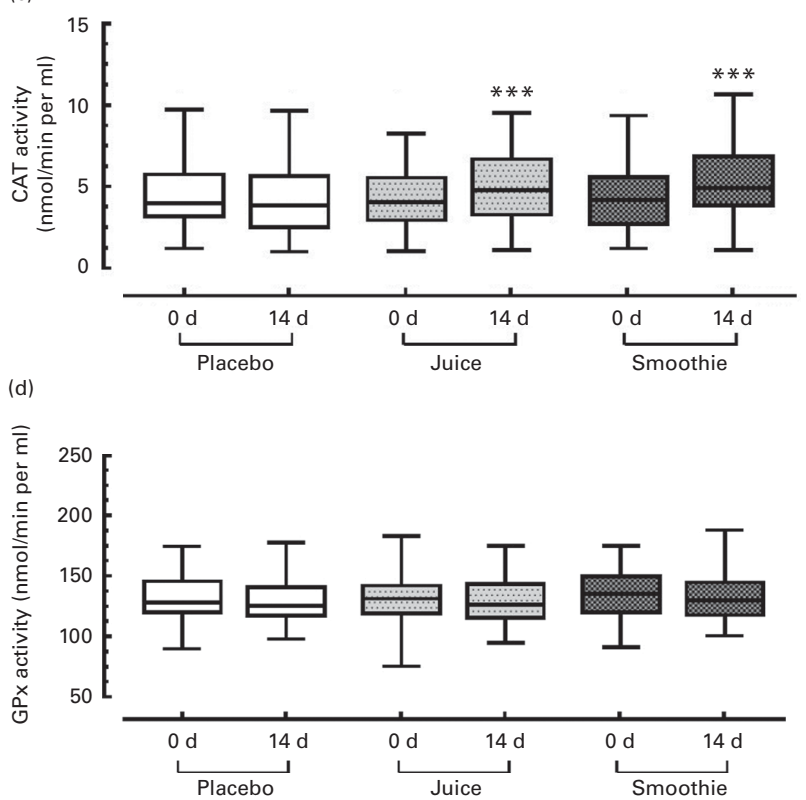

Fig. 2. Effects of beverage (placebo, juice and smoothie) intake on antioxidant enzymes before and after the intervention ( $n$ 30). The study participants consumed $330 \mathrm{ml}$ of placebo, juice and smoothie over $14 \mathrm{~d}$. Before $(0 \mathrm{~d})$ and after (14 d) the intervention, blood samples were drawn and activities of (a) superoxide dismutase (SOD) $)_{\text {plasma }}$, (b) SOD erythrocytes, (c) catalase (CAT) and (d) glutathione peroxidase (GPx) were measured in plasma or erythrocytes. Values are medians, with 25 th to 75 th percentiles represented by vertical bars. ${ }^{* \star}$ Median value was significantly different from that at baseline $(P<0 \cdot 001$; ANOVA, GLM (general linear mixed model)). 
cyanidin-3-glucoside, both these ACN are methylated. Although methylated $\mathrm{ACN}$ have a reduced antioxidant capacity in vitro, increased vasodilatation observed after the ingestion of a fruit/ vegetable juice could be attributed to methylated flavonoid metabolites ${ }^{(42,43)}$. On the other hand, using different methods for assessing the effects of ACN on target molecules could be a further point of interest. Weisel et al ${ }^{(30)}$ reported decreased oxidative DNA damage in peripheral blood mononuclear cells after the consumption of an ACN/polyphenol-rich fruit juice. Although the TEAC $(13.9 \mathrm{~mm})$ and ACN concentrations $(197.9 \mathrm{mg} / \mathrm{l})$ in the fruit juice were lower than those in the present study, the authors used the highly sensitive comet assay and determined reduced DNA damage even after a short-term intervention of only 1 week. However, they did not observe any changes in MDA concentrations during the 9-week intervention and assumed time-dependent reduction in the levels of lipid peroxidation markers.

Furthermore, it has to be mentioned that it is difficult to correlate antioxidative capacity with serum concentrations of parent ACN. Although intact ACN and their phase II conjugates (glucuronides or sulphates) might be present in the circulation, breakdown products from colonic fermentation (protocatechuic, hippuric, phenylacetic and phenyl propenoic acids) as well as conjugates of the breakdown products (protocatechuic glucuronide and protocatechuic sulphate) are present at much higher concentrations ${ }^{(44,45)}$. Using a ${ }^{13} \mathrm{C}$ tracer study with a single bolus of $500 \mathrm{mg}$ labelled cyanidin-3-glucoside, Czank et al. ${ }^{(46)}$ showed a 42 -fold higher abundance of ${ }^{13} \mathrm{C}$-labelled metabolites relative to $\left[{ }^{13} \mathrm{C}\right]$ cyanidin-3-glucoside at their maximum serum concentrations. Many of these metabolites also accumulated in the urine or were found in faeces. However, these metabolites could also exert antioxidative effects. In the present study, blood samples were drawn approximately $5 \mathrm{~h}$ after juice ingestion with malvidin-3-glucoside and peonidin-3-glucoside being found to be the most prominent circulating ACN. However, we could not conclude that the observed effects were only due to ACN. Using in vitro models, it has been revealed that syringic acid and 2,4,6trihydroxybenzaldehyde are the breakdown products of malvidin $^{(47)}$ and malvidin and vanillic acid are the breakdown products of peonidin ${ }^{(48)}$. Furthermore, concomitant with the results reported by Czank et al. ${ }^{(46)}$ using isotope-labelled cya-3-glucoside, numerous phenolic acids have been identified as the in vivo metabolites of ACN following blueberry consumption. For example, protocatechuic acid, a possible breakdown product of cyanidin-3-glucoside ${ }^{(49)}$, has been found to function as an antioxidant, not only due to its radical-scavenging effects but also due to its ability to influence antioxidant enzyme systems. It has been shown that increased GPx and glutathione reductase expression is induced by c-Jun NH2-terminal kinase-mediated phosphorylation of $\mathrm{Nrf2}^{(50-52)}$. In another study using oral supplementation of Medox ${ }^{\circledR}$ (Medox USA), a standardised ACN-rich supplement, plasma ACN concentrations were found to be high enough to affect the Nrf2-ARE pathways ${ }^{(19)}$. However, in the present study, the observed improvement in antioxidative capacity and reduced plasma and urinary TBARS levels could be a result of enhanced enzyme activities. 
The application of both juice and smoothie preparations revealed enhanced plasma CAT and SOD activities, but no influence on erythrocyte SOD activity after the $14 \mathrm{~d}$ intervention. Modulation of enzyme activity can occur indirectly through the modification of signal pathways as mentioned above or by direct competitive interactions possibly due to binding to the haem moiety or a protein region of $\mathrm{CAT}^{(53)}$. However, it has been shown that patients with CVD risk have reduced antioxidant enzyme (SOD and CAT) activities in serum with concomitantly enhanced TBARS levels ${ }^{(54)}$. Furthermore, if plasma SOD (extracellular SOD) activity is approximately 10-fold higher than cellular erythrocyte CuZn-SOD ${ }^{(55,56)}$ activity, ACN would be able to protect against oxidative stress and thus be beneficial not only for healthy individuals but also for patients at CVD risk. In this context, recently published data showed that the intake of freeze-dried blueberries with $375 \mathrm{mg}$ of ACN for 6 weeks in individuals with an increased cardiovascular risk reduced endogenously oxidised DNA bases and $\mathrm{H}_{2} \mathrm{O}_{2}$-induced DNA damage without affecting erythrocyte SOD activity ${ }^{(57)}$.

Endothelial dysfunction, besides the oxidative stress hypothesis, with changes in inflammatory parameters is considered to determine the first stages of AS. Emerging data have linked ROS generation with inflammatory markers. Furthermore, independent of other risk factors, low-grade inflammation with slightly enhanced secretion of cytokines or acute-phase proteins is predictive of CVD in healthy subjects $^{(58)}$. In the present study, ACN supplementation was found to have no effects on inflammatory markers in healthy young subjects. Neither inflammatory cytokines such as TNF- $\alpha$ and IL- 6 nor adhesion molecules such as integrins and acute-phase proteins (e.g. CRP) were affected under the experimental conditions in the present study. Only a few studies have already investigated the effects in healthy volunteers. Cherry consumption for $28 \mathrm{~d}$ was found to result in a decrease in CRP concentrations in healthy aged subjects $(45-60 \text { years })^{(59,60)}$, and the same effect was observed after the consumption of de-alcoholised red wine after a $20 \mathrm{~d}$ intervention $^{(61)}$. The consumption of Medox ${ }^{\circledR}$ by aged volunteers (40-75 years) for 3 weeks was found to be associated with a decrease in the concentrations of NF- $\mathrm{kB}$-regulated pro-inflammatory chemokines and immunoregulatory cytokines; a follow-up intervention with $330 \mathrm{ml}$ of blueberry juice per $\mathrm{d}$ for 4 weeks revealed decreased plasma concentrations of CRP and inflammatory cytokines ${ }^{(62)}$. Also, ingestion of freeze-dried grape powder over $30 \mathrm{~d}$ was found to slightly decrease soluble ICAM-1 concentrations with a concomitant decrease in blood pressure levels in patients with the metabolic syndrome ${ }^{(63)}$. The reason why ACN-rich beverages did not exert any effect in the present study could be the length of the intervention ( 2 weeks), the young age (mean 24.6 (SD 1.2) years) of the study population or the time point of blood sampling. Nevertheless, the time course of prevention strategies seems to be important, but studies with aged patients at risk and $\mathrm{ACN}$ are extremely rare. For example, an intake of blueberries (freeze-dried powder) over 6 weeks did not reveal any changes with respect to inflammatory biomarkers (TNF- $\alpha$ and CRP) in obese subjects throughout the 
study carried out by Stull et al. ${ }^{(64)}$. The consumption of cranberry juice ( $100 \mathrm{mg} \mathrm{ACN} / \mathrm{d}$, 4 weeks, $n$ 44) by patients with coronary artery risk factors or of grape juice $(965 \mathrm{mg}$ total polyphenols, 8 weeks, $n$ 17) by patients with hypertension was not found to alter plasma CRP or soluble ICAM-1 concentrations $^{(21)}$.

Taken together, although AS generates severe diseases primarily affecting middle-aged and old people, atherogenesis begins very early in life ${ }^{(65,66)}$. Therefore, primary prevention strategies should be implemented as early as possible. Thus, an evaluation of the functional capacity of $\mathrm{ACN}$ to prevent the onset of AS could be a useful strategy regarding the reduction of CVD risk. The participants of the present study were young, and the observed $\mathrm{ACN}$-induced antioxidative health benefits could be considered useful for the primary prevention of CVD. Comparisons of intervention studies with $\mathrm{ACN}$ are difficult due to variations in study designs, ACN patterns, dosages and preparations as well as differences in the polyphenol content of the general diet during the study. Although the observed effects on antioxidative markers can be attributed to ACN, it should be kept in mind that the ACN-rich juice and smoothie contained additional polyphenols such as polymeric ACN and simple phenolic acids such as protocatechuic acid and chlorogenic acid, which have also been shown to have the potential to influence antioxidative and anti-inflammatory parameters in vitro and in vivo ${ }^{(67-69)}$.

The strength of the present study was the homogeneous study population ( $n$ 30) and its cross-over design that permitted the same participants to receive all treatments, which minimised interferences of possible confounding variables. Division of the study population into six groups with different treatment sequences minimised carry-over effects. Furthermore, the application of an ACN-depleted placebo juice as a control beverage and dietary restrictions of polyphenol intake without affecting basal activities was a further important point in the study design. A limitation of the study was that the volunteers were healthy without indications of CVD events, which made it difficult to detect an effect on inflammationassociated events in atherogenesis. In further studies, emphasis should be placed on the identification and characterisation of $\mathrm{ACN}$ and their metabolites in plasma, which would provide information on metabolites responsible for the effects of ACN. In conclusion, the present study demonstrates that due to their potent antioxidant activities, ingestion of ACN-rich beverages could be a useful strategy to reduce the risk of CVD.

\section{Acknowledgements}

The authors cordially thank the study participants. They also thank Cordula Becker, Nadine Metz, Sina Streichert, Nicole Tscherney and Marcel Zoremba for their excellent technical support in performing blood and urine sample analyses.

The present study was funded by the German Ministry of Education and Research (BMBF) as part of the research consortium ANTHONIA (grant no. 0315379A). The BMBF had no role in the design and analysis of the study or in the writing of this article.
The authors' contributions are as follows: S. K., S. R. and C. K. were the principal investigators of the study and responsible for the study design and wrote the manuscript; G. A. and $\mathrm{C}$. H. B. contributed to the study design, intervention and sample handling; B. F. and H. D. prepared the ACN-rich and ACN-depleted beverages and measured ACN content; J. H. was responsible for the statistical analyses. All authors approved the final version of the manuscript.

None of the authors has any conflicts of interest to declare.

\section{References}

1. World Health Organization (2013) The World Health Report 2008. http://www.who.int/whr/en/ (accessed 1 July 2013).

2. Badimon L \& Vilahur G (2012) LDL-cholesterol versus HDL-cholesterol in the atherosclerotic plaque: inflammatory resolution versus thrombotic chaos. Ann N Y Acad Sci 1254, $18-32$.

3. Hansson KG (2005) Inflammation, atherosclerosis, and coronary artery disease. $N$ Engl J Med 352, 1685-1695.

4. Dadu RT, Nambi V \& Ballantyne CM (2012) Developing and accessing cardiovascular biomarkers. Transl Res 159, 265-276.

5. Fruchart JC, Nierman MC, Stroes ES, et al. (2004) New risk factors for atherosclerosis and patient risk assessment. Circulation 109, 15-19.

6. Badimon L, Storey RF \& Vilahur G (2011) Update on lipids, inflammation and atherothrombosis. Thromb Haemost 105 , S34-S42.

7. Ishigami N, Isoda K \& Adachi T (2011) Deficiency of CuZn superoxide dismutase promotes inflammation and alters medial structure following vascular injury. J Atheroscler Thromb 18, 1009-1017.

8. de la Sierra A \& Larrousse M (2010) Endothelial dysfunction is associated with increased levels of biomarkers in essential hypertension. J Hum Hypertens 24, 373-379.

9. Laurila JP, Laatikainen LE, Castellone MD, et al. (2009) SOD3 reduces inflammatory cell migration by regulating adhesion molecule and cytokine expression. PLOS ONE 4, e5786.

10. Kwon MJ, Jeon YJ, Lee KY, et al. (2012) Superoxide dismutase 3 controls adaptive immune responses and contributes to the inhibition of OVA-induced allergic airway inflammation in mice. Antioxid Redox Signal 15, 1376-1392.

11. Schaller G, Dittrich P, Felizeter M, et al. (2012) Human pharmacokinetics of intravenous recombinant human $\mathrm{Cu} / \mathrm{Zn}$ superoxide dismutase. Int J Clin Pharmacol Ther 50, 413-417.

12. Siasos G, Tousoulis D, Tsigkou V, et al. (2013) Flavonoids in atherosclerosis: an overview of their mechanisms of action. Curr Med Chem 20, 2641-2660.

13. Kim SM, Chung MJ, Ha TJ, et al. (2012) Neuroprotective effects of black soybean anthocyanins via inactivation of ASK1-JNK/p38 pathways and mobilization of cellular sialic acids. Life Sci 6, 874-882.

14. Alvarez-Suarez JM, Dekanski D, Ristić S, et al. (2011) Strawberry polyphenols attenuate ethanol-induced gastric lesions in rats by activation of anti-oxidant enzymes and attenuation of MDA increase. PLOS ONE 6, e25878.

15. Yi L, Chen CY, Jin X, et al. (2012) Differential suppression of intracellular reactive oxygen species-mediated signaling pathway in vascular endothelial cells by several subclasses of flavonoids. Biochemie 94, 2035-2044

16. Paur I, Austenaa LM \& Blomhoff R (2008) Extracts of dietary plants are efficient modulators of nuclear factor kappa B. Food Chem Toxicol 46, 1288-1297. 
17. Nguyen T, Nioi P \& Pickett CB (2009) The Nrf2-antioxidant response element signaling pathway and its activation by oxidative stress. J Biol Chem 284, 13291-13295.

18. Lee JM \& Johnson JA (2004) An important role of Nrf2-ARE pathway in the cellular defense mechanism. J Biochem Mol Biol 37, 139-143.

19. Cimino F, Speciale A, Anwar S, et al. (2013) Anthocyanins protect human endothelial cells from mild hyperoxia damage through modulation of Nrf2 pathway. Genes Nutr 8, 391-399.

20. Boettler U, Sommerfeld K, Volz N, et al. (2011) Coffee constituents as modulators of $\mathrm{Nrf} 2$ nuclear translocation and ARE (EpRE)-dependent gene expression. J Nutr Biochem 22, 426-440.

21. Dohadwala MM, Holbrook M, Hamburg NM, et al. (2011) Effects of cranberry juice consumption on vascular function in patients with coronary artery disease. Am J Clin Nutr 93, 934-940.

22. Triebel S, Trieu HL \& Richling E (2012) Modulation of inflammatory gene expression by a bilberry (Vaccinium myrtillus L.) extract and single anthocyanins considering their limited stability under cell culture conditions. J Agric Food Chem 12, 8902-8910.

23. Hassimotto NM, Pinto Mda S \& Lajolo FM (2008) Antioxidant status in humans after consumption of blackberry (Rubus fruticosus L.) juices with and without defatted milk. J Agric Food Chem 24, 11727-11733.

24. Kolehmainen M, Mykkänen O, Kirjavainen PV, et al. (2012) Bilberries reduce low-grade inflammation in individuals with features of metabolic syndrome. Mol Nutr Food Res 56, 1501-1510.

25. de Mello VD, Schwab U, Kolehmainen M, et al. (2011) A diet high in fatty fish, bilberries and wholegrain products improves markers of endothelial function and inflammation in individuals with impaired glucose metabolism in a randomised controlled trial: the Sysdimet study. Diabetologia 54, 2755-2767.

26. Karlsen A, Paur I, Bøhn SK, et al. (2010) Blueberry juice modulates plasma concentration of NF- $\mathrm{KB}$ related inflammatory markers in subjects at increased risk of CVD. Eur J Nutr 49, 345-355.

27. Fröhling B, Patz C, Dietrich H, et al. (2012) Anthocyanins, total phenolics and antioxidant capacities of commercial red grape juices, black currant and sour cherry nectars. Fruit Process 3, 100-104.

28. Spormann TM, Albert FW, Rath T, et al. (2008) Anthocyanin/ polyphenolic-rich fruit juice reduces oxidative cell damage in an intervention study with patients on hemodialysis. Cancer Epidemiol Biomarkers Prev 17, 3372-3380.

29. Graumlich JF, Ludden TM, Conry-Cantilena C, et al. (1997) Pharmacokinetic model of ascorbic acid in healthy male volunteers during depletion and repletion. Pharm Res 14, $1133-1139$

30. Weisel T, Baum M, Eisenbrand G, et al. (2006) An anthocyanin/polyphenolic-rich fruit juice reduces oxidative DNA damage and increases glutathione level in healthy probands. Biotechnol J 1, 388-397.

31. Duthie SJ, Jenkinson AMcE, Crozier A, et al. (2006) The effects of cranberry juice consumption on antioxidant status and biomarkers relating to heart disease and cancer in healthy human volunteers. Eur J Nutr 45, 113-122.

32. Miller NJ \& Rice-Evans C (1997) Factors influencing the antioxidant activity determined by the ABTS + radical cation assay. Free Radic Res 26, 195-199.

33. Miller NJ, Rice-Evans C, Davies MJ, et al. (1993) A novel method for measuring antioxidant capacity and its application to monitoring the antioxidant status in premature neonates. Clin Sci (Lond) 84, 407-412.

34. Rice-Evans C \& Miller N (1994) Total antioxidant status in plasma and body fluids. Methods Enzymol 234, 279-293.

35. Helger R, Rindfrey H \& Hilgenfeldt J (1974) (Direct estimation of creatinine in serum and in urine without deproteinization using a modified Jaffé method). Z Klin Chem Klin Biochem 12, 344-349.

36. Peake M \& Whiting M (2006) Measurement of serum creatinine - current status and future goals. Clin Biochem Rev 27, 173-184.

37. Seelig H \& Wüst H (1969) Die Kreatininbestimmung mit der Jaffé Reaktion (The Jaffe reaction with creatinine. The reaction product and general reaction conditions). Ärztl Lab 15, 34-39.

38. Lowry OH, Lopez JA \& Bessey OA (1945) The determination of ascorbic acid in small amounts of serum. J Biol Chem $\mathbf{1 6 0}$ 609-615.

39. Omaye ST, Turnbull JD \& Sauberlich HE (1979) Selected methods for the determination of ascorbic acid in animal cells, tissues, and fluids. Methods Enzymol 62, 3-11.

40. Watzl B, Kulling SE, Möseneder J, et al. (2005) A 4-wk intervention with high intake of carotenoid-rich vegetables and fruit reduces plasma C-reactive protein in healthy, nonsmoking men. Am J Clin Nutr 82, 1052-1058.

41. Del Rio D, Borges G \& Crozier A (2010) Berry flavonoids and phenolics: bioavailability and evidence of protective effects. Br J Nutr 104, 67-90.

42. George TW, Waroonphan S, Niwat C, et al. (2012) Effects of acute consumption of a fruit and vegetable purée-based drink on vasodilation and oxidative status. Br J Nutr 109, 1442-1452.

43. Kuhnle G, Spencer JPE \& Schroeter H (2000) Epicatechin and catechin are $O$-methylated and glucuronidated in the small intestine. Biochem Biophys Res Commun 277, 507-512.

44. Koli R, Erlund I, Jula A, et al. (2010) Bioavailability of various polyphenols from a diet containing moderate amounts of berries. J Agric Food Chem 58, 3927-3932.

45. Harini R \& Pugalendi KV (2010) Antioxidant and antihyperlipidaemic activity of protocatechuic acid on streptozotocindiabetic rats. Redox Rep 15, 71-80.

46. Czank C, Cassidy A, Zhang Q, et al. (2013) Human metabolism and elimination of the anthocyanin, cyanidin-3-glucoside: a (13)C-tracer study. Am J Clin Nutr 97, 995-1003.

47. Forester SC \& Waterhouse AL (2008) Identification of Cabernet Sauvignon anthocyanin gut microflora metabolites. J Agric Food Chem 56, 9299-9304.

48. Labib S, Erb A, Kraus M, et al. (2004) The pig caecum model: a suitable tool to study the intestinal metabolism of flavonoids. Mol Nutr Food Res 48, 326-332.

49. Vitaglione P, Donnarumma G, Napolitano A, et al. (2007) Protocatechuic acid is the major human metabolite of cyanidin-glucosides. J Nutr 137, 2043-2048.

50. Varì R, D'Archivio M, Filesi C, et al. (2011) Protocatechuic acid induces antioxidant/detoxifying enzyme expression through JNK-mediated Nrf2 activation in murine macrophages. J Nutr Biochem 22, 409-417.

51. Kropat C, Mueller D, Boettler U, et al. (2013) Modulation of Nrf2-dependent gene transcription by bilberry anthocyanins in vivo. Mol Nutr Food Res 57, 545-550.

52. Masella R, Santangelo C, D'Archivio M, et al. (2012) Protocatechuic acid and human disease prevention: biological activities and molecular mechanisms. Curr Med Chem 19 2901-2917. 
53. Doronicheva N, Yasui H \& Sakurai H (2007) Chemical structure-dependent differential effects of flavonoids on the catalase activity as evaluated by a chemiluminescent method. Biol Pharm Bull 30, 213-217.

54. Kumar EP, Mukherjee R, Senthil R, et al. (2012) Evaluation of oxidative stress and antioxidant status in patients with cardiovascular disease in rural populations of the Nilgiris, South India. ISRN Pharmacol 2012, 941068.

55. Karlsson K \& Markl SL (1988) Extracellular superoxide dismutase in the vascular system of mammals. Biochem $J$ 255, 223-228.

56. Strålin P, Karlsson K, Johansson BO, et al. (1995) The interstitium of the human arterial wall contains very large amounts of extracellular superoxide dismutase. Arterioscler Thromb Vasc Biol 15, 2032-2036.

57. Riso P, Klimis-Zacas D, Del Bo' C, et al. (2013) Effect of a wild blueberry (Vaccinium angustifolium) drink intervention on markers of oxidative stress, inflammation and endothelial function in humans with cardiovascular risk factors. Eur J Nutr 52, 949-961.

58. Koenig W \& Wanner C (1999) C-reactive protein and coronary artery disease - what is the link? Nephrol Dial Transplant 14, 2798-2800.

59. Kelley DS, Rasooly R, Jacob RA, et al. (2006) Consumption of bing sweet cherries lowers circulating concentrations of inflammation markers in healthy men and women. $J$ Nutr 136, 981-986.

60. Kelley DS, Adkins Y, Reddy A, et al. (2013) Sweet bing cherries lower circulating concentrations of markers for chronic inflammatory diseases in healthy humans. $J$ Nutr 143, 340-344.

61. Queipo-Ortuño MI, Boto-Ordóñez M, Murri M, et al. (2012) Influence of red wine polyphenols and ethanol on the gut microbiota ecology and biochemical biomarkers. Am J Clin Nutr 95, 1323-1334

62. Karlsen A, Retterstol L, Laake P, et al. (2007) Anthocyanins inhibit nuclear factor-B activation in monocytes and reduce plasma concentrations of pro-inflammatory mediators in healthy adults. J Nutr 137, 1951-1954.

63. Barona J, Aristizabal JC, Blesso CN, et al. (2012) Grape polyphenols reduce blood pressure and increase flow-mediated vasodilation in men with metabolic syndrome. J Nutr 142, $1626-1632$

64. Stull AJ, Cash KC, Johnson WD, et al. (2010) Bioactives in blueberries improve insulin sensitivity in obese, insulinresistant men and women. J Nutr 140, 1764-1768.

65. Morrison KM, Atkinson SA, et al. (2009) The Family Atherosclerosis Monitoring In earLY life (FAMILY) study: rationale, design, and baseline data of a study examining the early determinants of atherosclerosis. Am Heart $J \mathbf{1 5 8}$, 533-539

66. Napoli C, Infante T \& Casamassimi A (2011) Maternal-foetal epigenetic interactions in the beginning of cardiovascular damage. Cardiovasc Res 92, 367-374.

67. Shi H, Dong L, Dang X, et al. (2013) Effect of chlorogenic acid on LPS-induced proinflammatory signaling in hepatic stellate cells. Inflamm Res 62, 581-587.

68. Esposito D, Chen A, Grace MH, et al. (2014) Inhibitory effects of wild blueberry anthocyanins and other flavonoids on biomarkers of acute and chronic inflammation in vitro. J Agric Food Chem 62, 7022-7028.

69. Volz N, Boettler U, Winkler S, et al. (2012) Effect of coffee combining green coffee bean constituents with typical roasting products on the Nrf2/ARE pathway in vitro and in vivo. J Agric Food Chem 60, 9631-9641. 